\section{VIDEO REVIEW}

Painful Procedures: Helping Children to Cope. Paediatric advice, literature and support; 1994. Price $£ 50$ (includes postage and packing). Available from Marianne Flint, Paediatric Renal Unit, Nottingham City Hospital, Hucknall Road, Nottingham NG5 1 PB.

This is presented in the form of a package. The package contains a video, several accompanying booklets and a complementary manual which provides additional information.

The video aims to show parents and staff ways in which children can be helped to cope with painful procedures, such as venepuncture. It lasts for approximately 10 minutes, and demonstrates techniques of distraction. It also gives the viewer tips on how to empower the child during such procedures. However, I feel the video could be a source of anxiety to many parents as it shows two extremely distressed children having venepuncture performed. It also shows older children sitting alone with the doctor or phlebotomist having bloods taken. At this age children are usually able to understand why such procedures are necessary, and, as is pointed out, they may be offered choices, for example, from which arm the blood should be taken.

Children's nurses and doctors frequently witness toddlers being restrained by a parent or nurse during these procedures. No toddler likes to be held or restrained especially when ill. It is vital for such children that the procedure is over quickly, as pain free as possible (use of EMLA cream), and that a family face is there to comfort them and offer reassurance. Unfortunately the video gives no explanation about why toddlers protest and no reassurance to parents that this is normal behaviour and why their presence is important to their child.

The manual discusses the case for preparing children for painful procedures. This area, I feel, was particularly pertinent to nurses. It demonstrates that by taking time and trouble to address the issue the initial time and effort will be recouped in the long run to the benefit of the staff, parents and, most of all, children.

The use of play specialists and trained childrens nurses is vital in paediatrics - this is acknowledged in the text. Play is also vital in the child's development - the manual describes simply ways of play preparation and role play for children of 2 years and above. All the ideas are viable and could be easily used in the ward at little or no cost.

The ideas in the package are not new, but people dealing with children do need reminding of their importance and how painful procedures affect staff, parents, and children. I feel this package could be used as part of an orientation programme for nurses, doctors, and students.

It needs to include acknowledgment of the special needs of toddlers, as this age group is sadly neglected despite being a high percentage of our hospital admissions.

SUE WATSON Senior nurse manager in paediatrics

\title{
Melioidosis
}

Here's one to store in your cerebral instant access account in case you're ever tempted to take up practice in South East Asia or northern Australia, or come across a sick child recently arrived from those parts, where melioidosis is endemic. It is caused by Pseudomonas pseudomallei which inhabits soil and water and infects people usually through either the skin or the lungs.

Only some 10 to $17 \%$ of those affected are children, but a recent report from north east Thailand (Pagakrong Lumbiganon and Surapon Viengnondha, Pediatric Infectious Disease fournal 1995; 14: 136-40) gives details of 55 children aged 9 months to 14 years seen over a period of 13.5 years. Thirty five had localised infections and 20 septicaemia.

The most common form of localised infection was an acute bilateral suppurative parotitis. Other sites affected, usually with abscess formation, were skin, lymph nodes of head and neck, lung and pleural cavity, brain, and cerebrospinal fluid shunt. All of these patients recovered.

Multiple organ involvement (lung, skin, spleen, liver, and skeletal) was common in those with septicaemia and 12 of the 20 died. Underlying chronic disease such as diabetes, leukaemia, asplastic anaemia, chronic renal failure, or nephrotic syndrome was often a feature of the septicaemic form.

Antibiotic treatment for the septicaemic disease was with cotrimoxazole and kanamycin with or without doxycycline (eight patients), ceftazidime with or without co-trimoxazole (four patients), and imipenem (four patients). Localised melioidosis was usually treated with either co-trimoxazole or doxycycline, although one patient each received imipenem and ciprofloxacin.

Think South East Asia, think northern Australia, think septicaemic shock, think peculiar abscess formation or suppurative parotitis: think melioidosis. 